\title{
Adaptividade de Malhas na Aproximação Lagrangeana de Leis de Conservação
}

S. MANCUSO ${ }^{1}$, F. PEREIRA ${ }^{2}$, G. de SOUZA ${ }^{3}$, LABTRAN, Departamento de Modelagem Computacional, IPRJ, UERJ, 28630-050 Nova Friburgo, RJ, Brasil.

\begin{abstract}
Resumo. Métodos eficientes para a resolução de equações de transporte convectivo constituem uma ferramenta importante na modelagem computacional de problemas de engenharia como, por exemplo, o escoamento de fluidos em meios porosos. Neste trabalho é apresentado um novo método numérico para a resolução de leis de conservação escalares que utiliza técnicas lagrangeanas e adaptividade da malha computacional, que foi chamado de DSTC ("Dynamical Space Time Coarsening"). Este método faz uso da identidade de conservação local que aparece em $[1,3,4]$. Tal metodologia foi aplicada na aproximação numérica das equações de Burgers e Buckley-Leverett, sendo esta última utilizada na modelagem de escoamentos bifásicos em reservatórios de petróleo. A técnica apresentou bom desempenho computacional, precisão na captura de saltos, ausência de oscilações espúrias e convergência numérica sob refinamento da malha computacional.
\end{abstract}

\section{Introdução}

As leis de conservação aparecem freqüentemente na modelagem matemática de diversos fenômenos físicos. Como exemplo, pode-se citar problemas de transporte convectivo não linear, como a propagação de choques e os escoamentos bifásicos em meios porosos. Estes são especialmente importantes na exploração de petróleo.

Neste trabalho foi desenvolvido um esquema numérico que utiliza uma estratégia lagrangeana localmente conservativa e a evolução da solução numérica para malhas não-uniformes. Além disso, utiliza-se uma combinação de células para permitir passos de tempo maiores e, conseqüentemente, reduzir a suavização numérica presente nas soluções. Através da observação dos resultados de simulações numéricas se nota que as técnicas localmente conservativas são bastante adequadas para o tratamento de leis de conservação hiperbólicas e que o novo esquema proposto apresenta resultados bastante precisos para o problema de propagação de choques.

\section{Esquema Numérico}

A seguir é apresentado o método numérico desenvolvido neste trabalho, que tem sua construção baseada no método presente em [3]. O esquema aqui proposto utiliza

\footnotetext{
${ }^{1}$ smancuso@iprj.uerj.br

${ }^{2}$ pereira@iprj.uerj.br

3 ev_grazione@iprj.uerj.br
} 
tubos integrais no espaço-tempo na forma explícita. Para a elaboração do método, considere uma lei de conservação hiperbólica em uma dimensão espacial, como a mostrada abaixo:

$$
u_{t}+f(u)_{x}=0,
$$

ou na sua forma divergente

$$
\nabla_{t, x} \cdot\left(\begin{array}{c}
u \\
f(u)
\end{array}\right)=0 \quad x \in \mathbb{R}, \quad \text { com condição inicial } \quad u(x, 0)=u_{0}(x) .
$$

\subsection{Evolução temporal}

Para a evolução temporal as variáveis discretizadas calculadas no tempo $t^{n}$ são denotadas acrescentando-lhes o índice superior $n$. Considera-se para a equação (2.1) uma discretização espacial em malha original uniforme de comprimento $\Delta x$ para cada célula. Define-se $h_{j}^{n}$ o tamanho da célula $j$ no tempo $t^{n}, x_{j}^{n}$ representa o centro da j-éssima célula no mesmo instante onde $j$ é um inteiro, e $x_{j-1 / 2}^{n}$ e $x_{j+1 / 2}^{n}$, os seus vértices. Utilizando para a evolução temporal os tubos e curvas integrais expostos na seção 2.2 de [5] e seguindo o raciocínio ali apresentado, define-se $U_{j}^{n}$ como a discretização constante por partes de $u$ no instante $t^{n}$

$$
U_{j}^{n}(t)=\frac{1}{h_{j}^{n}} \int_{x_{j-1 / 2}^{n}}^{x_{j+1 / 2}^{n}} u(x, t) d x
$$

com $x_{j-1 / 2}^{n}=y\left(x_{j}^{n-1}, t^{n}\right)$ onde $\frac{d y}{d t}=\frac{f\left(U_{j}^{n-1}\right)}{U_{j}^{n-1}}$ e $y\left(x_{j}^{n-1}, t^{n-1}\right)=x_{j}^{n-1}$. O valor de $U_{j}^{n+1}$ em cada intervalo $\left[x_{j-1 / 2}^{n+1}, x_{j+1 / 2}^{n+1}\right), j \in \mathbb{Z}$, da nova malha é dado por

$$
\begin{aligned}
U_{j}^{n+1} & =\frac{1}{h_{j}^{n+1}}\left(\int_{x_{j}^{n}}^{x_{j+1 / 2}^{n}} U\left(x, t^{n}\right) d x+\int_{x_{j+1 / 2}^{n}}^{x_{j+1}^{n}} U\left(x, t^{n}\right) d x\right) \\
& =\frac{1}{h_{j}^{n+1}}\left(\frac{h}{2} U_{j}^{n}+\frac{h}{2} U_{j+1}^{n}\right) .
\end{aligned}
$$

Assim determina-se $U^{n+1}$ e o processo se repete até o fim da simulação.

\subsection{Aproximação linear por partes}

A precisão do método pode ser aumentada pela substituição da função contante por partes $U(x, t)$ por uma função linear por partes $L(x, t)$. Para isto, deve ser obtida uma inclinação $U_{j}^{\prime}(t)$ relacionada à célula de centro $x_{j}^{n}$. Através de um tipo de MinMod (Módulo Mínimo; ver [2]) obtém-se a derivada numérica espacial

$$
\frac{\Delta U_{j}^{n}}{h_{j}^{n}}=\operatorname{modmin}\{a, b, c\} a /|a|
$$


onde

$$
a=\frac{U_{j}^{n}-U_{j-1}^{n}}{\left(h_{j-1}^{n}+h_{j}^{n}\right) 0,5}, b=\frac{U_{j+1}^{n}-U_{j-1}^{n}}{\left(h_{j-1}^{n}+2 h_{j}^{n}+h_{j+1}^{n}\right) 0,5}, c=\frac{U_{j+1}^{n}-U_{j}^{n}}{\left(h_{j}^{n}+h_{j+1}^{n}\right) 0,5}
$$

e min é a função mínimo. Usando os valores $\Delta U_{j}^{n}(t)$, constrói-se então a discretização linear por partes mostrada pela equação $(2.7)$, que preserva a conservação local

$$
L_{j}\left(x, t^{n}\right)=U_{j}^{n}+\left(x-x_{j}^{n}\right) \frac{\Delta U_{j}^{n}}{h_{j}^{n}}, \quad x_{j-1 / 2}^{n}<x \leq x_{j+1 / 2}^{n} .
$$

Neste caso, a equação (2.4) toma a seguinte forma

$$
U_{j}^{n+1}=\frac{1}{h_{j}^{n+1}}\left(\int_{x_{j}^{n}}^{x_{j+1 / 2}^{n}} L\left(x, t^{n}\right) d x+\int_{x_{j+1 / 2}^{n}}^{x_{j+1}^{n}} L\left(x, t^{n}\right) d x\right) .
$$

Nesta implementação segue-se o raciocínio análogo ao utilizado para resolver (2.4). A metodologia foi denominada pelos autores de DSTC-R ("Dynamical SpaceTime Coarsening with Reconstruction"). Com uso da reconstrução, as curvas integrais precisam de uma melhor aproximação dos $x_{j}^{n+1}$. A Figura 1 mostra a discretização linear por partes.

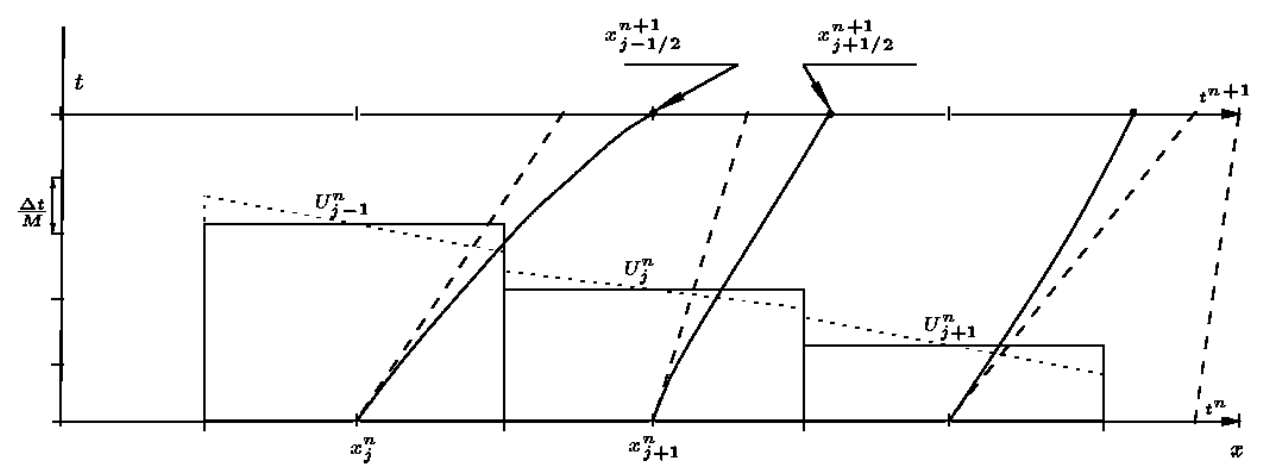

Figura 1: Linhas tracejadas correspondem às tangentes à curva integral no tempo $t^{n}$, para o caso sem correção, enquanto linhas contínuas mostram tubos corrigidos. As linhas pontilhadas apresentam a reconstrução linear $L_{j}$, feita a partir do MINMOD.

Neste caso, as curvas são construídas por $M$ passos: no primeiro, constrói-se a curva da forma convencional com um avanço de tempo $\Delta t / M$ usando $U_{j}^{n}$ indo de $x_{j}^{n}$ até $x_{j, 1}^{n}$. Para calcular $x_{j, i+1}^{n}$ calcula-se $U_{j, i}^{n}$ em $\left(x_{j, i}^{n}, t^{n}+i \Delta t / M\right)$ por expansão em série de Taylor no tempo, sobre $L_{j}\left(x_{j, i}^{n}, t^{n}\right)$, obtendo-se $x_{j, i+1}^{n}=x_{j, i}^{n}+\frac{f\left(U_{j, i}^{n}\right)}{U_{j, i}^{n}} \frac{\Delta t}{M}$. Isto é feito enquanto $i<M$ (até completar o $\Delta t$ total permitido). Uma vez determinados os valores de $U$ no instante $t+\Delta t$, repete-se o procedimento partindo, agora, dos centros das células da nova malha não-uniforme para determinar os $x^{n+2}$. A Figura 1 também mostra a correção dos tubos. 


\subsection{Restrição no passo de tempo}

A evolução temporal utiliza um incremento $\Delta t$ que não permita que a solução dos problemas de Riemann oriundos dos vértices das células atinjam os centros das mesmas. O passo de tempo $\Delta t$ deve satisfazer uma condição do tipo CFL e assume valores dinamicamente durante a simulação (faz-se uso do menor tamanho de célula da malha, $\left.h_{\min }\right)$. Tem-se a restrição

$$
\max \left\{f_{\text {sup }}^{\prime}-\left(\frac{f(U)}{U}\right)_{\text {sup }},\left(\frac{f(U)}{U}\right)_{\text {inf }}-f_{\text {inf }}^{\prime}\right\} \frac{\Delta t^{n}}{h_{\text {min }}} \leq \frac{1}{2},
$$

onde max é a função máximo e

$$
\begin{gathered}
f_{\text {sup }}^{\prime}=\sup \left\{f^{\prime}(U) \mid U \in \mathbb{D}_{f^{\prime}}\right\} \text { e } f_{\text {inf }}^{\prime}=\inf \left\{f^{\prime}(U) \mid U \in \mathbb{D}_{f^{\prime}}\right\} \\
\left(\frac{f(U)}{U}\right)_{\text {sup }}=\sup \left\{\left(\frac{f(U)}{U}\right) \mid U \in \mathbb{D}_{f}\right\} \text { e }\left(\frac{f(U)}{U}\right)_{\text {inf }}=\inf \left\{\left(\frac{f(U)}{U}\right) \mid U \in \mathbb{D}_{f}\right\}
\end{gathered}
$$

\section{Combinação de Células}

Dependendo da função de fluxo $f$ que aparece na equação (2.1), o passo de tempo pode tender a uma queda acentuada no decorrer da simulação. Por isso, se combinam dinamicamente células de tamanho inferior a um valor crítico para formar células maiores usando uma técnica denominada DSTC ("Dynamical Space Time Coarsening"). A idéia é construir os tubos partindo apenas de centros de células pré-selecionadas a fim de evitar o surgimento de células que causem refinamento excessivo da malha. O cálculo do escalar $U$ médio continua sendo feito usando a quantidade contida entre as paredes dos tubos. O tamanho crítico é uma fração do tamanho de célula na malha original e foi denominada fator crítico, simbolizado por $f c$.

Quando uma determinada célula alcança um tamanho crítico faz-se uma combinação entre esta e uma de suas vizinhas, formando uma célula maior com uma novo valor de $U$ médio determinado pela soma das quantidades $h_{j} U_{j}$ de cada uma. A combinação ocorre entre a célula que ficou muito reduzida e a sua vizinha com valor de $U$ mais próximo: se em um passo de tempo, por exemplo, se a célula $\mathrm{j}+1$ apresenta um tamanho inferior ao valor crítico com $U$ mais próximo ao da célula $\mathrm{j}$ do que ao da célula $\mathrm{j}+2$, combinam-se as células $\mathrm{j}+1 \mathrm{e} j$, criando uma nova célula $\mathrm{j}$. Por outro lado, se ocorrer a combinação das células $j+1$ com $j+2$ forma-se uma nova célula $\mathrm{j}+1$. Tal processo é repetido até que todas as células tenham tamanho maior do que o crítico. A seguir calcula-se o centro dessas novas células e determina-se em que células da malha antes da pré-seleção encontram-se esses pontos médios. A evolução temporal é idêntica à que foi apresentada anteriormente e será realizada sobre a malha anterior à pré-seleção, mas não serão construídos os tubos que partiriam daquelas células que foram combinadas na pré-seleção, mas que não contém os pontos médios das células resultantes da combinação, como é apresentado na Figura 2. 


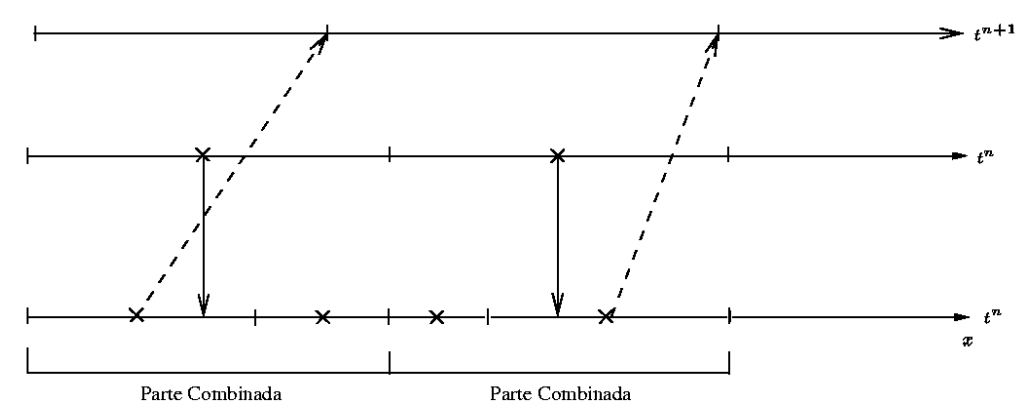

Figura 2: Combinação de células: linhas contínuas mostram em que ponto está o centro de uma célula combinada em relação as células que a formaram e linhas tracejadas mostram de onde vão partir os tubos.

\section{Simulações Numéricas}

Nos experimentos numéricos utilizou-se uma condição de injeção lateral na fronteira esquerda da malha computacional correspondente ao fluxo associado a condição inicial à esquerda do problema de Riemann (ver detalhes em [3]). O fator crítico $f c$ utilizado foi de 0,2 e o valor $M$ de passos para construção dos tubos integrais foi igual a 10, em todas as simulações.

Na equação de Burgers a função $f$ da equação (2.1) é

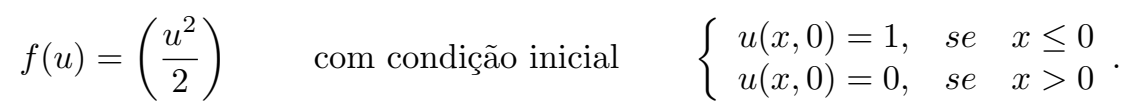

Nos testes para esta equação o comprimento da região física foi de $12800 \mathrm{~cm}$ e o tempo de simulação de $15000 \mathrm{~s}$. As Figuras 3 e 4 apresentam um estudo de refinamento de malha e a comparação da solução numérica em 256 células com a solução analítica para o DSTC-R, respectivamente.

As simulações numéricas conseguiram representar o salto com velocidade de propagação correta, como pode ser visto na comparação com a solução analítica. Além disso, a estratégia DSTC-R produziu resultados com baixa difusão numérica e pôde-se observar que em uma malha de 128 células atingiu-se um bom nível de convergência numérica.

Para a Eq. de Buckley-Leverett, a função de fluxo $f$ é

$$
f(s)=\lambda_{w}(s) v \quad \operatorname{com} \quad\left\{\begin{array}{l}
\lambda_{w}(s)=\frac{k_{r_{w}}(s)}{\mu_{w} \lambda(s)} \\
\lambda(s)=\frac{k_{r_{o}}(s)}{\mu_{o}}+\frac{k_{r_{w}}(s)}{\mu_{w}} \\
k_{r_{o}}(s)=\left(1-\left(1-s_{r_{o}}\right)^{-1} s\right)^{2} \\
k_{r_{w}}(s)=\left(1-s_{r_{w}}\right)^{-2}\left(s-s_{r_{w}}\right)^{2}
\end{array}\right.
$$

onde $\mathrm{w}=$ água e $\mathrm{o}=$ óleo. Para a fase $\alpha(\alpha=w, o)$ tem-se: $\lambda_{\alpha}(s)$, mobilidade; $k_{r_{\alpha}}(s)$, permeabilidade relativa; $s_{r_{\alpha}}$, saturação residual e $\mu_{\alpha}$, a viscosidade. Os demais termos são: $\lambda(s)$, mobilidade total do sistema e $v$, a velocidade do fluido (velocidade 


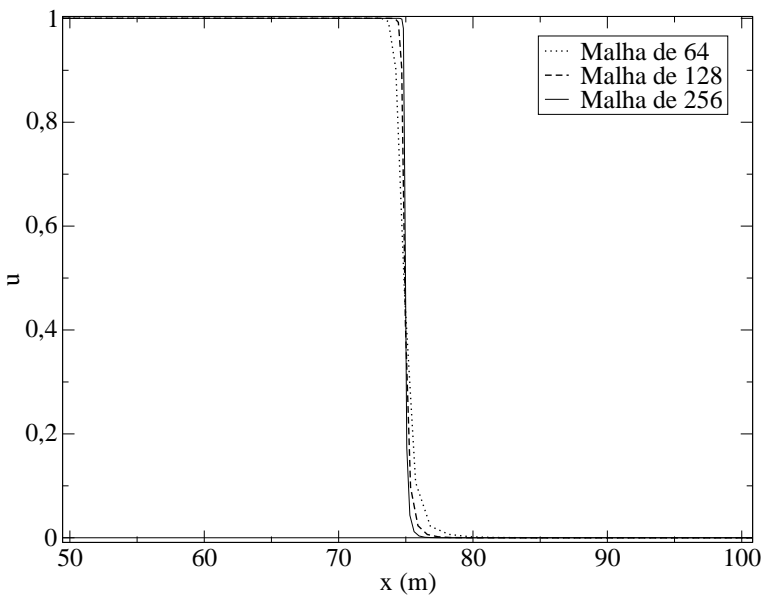

Figura 3: Eq. de Burgers: refinamento de malha (DSTC-R).

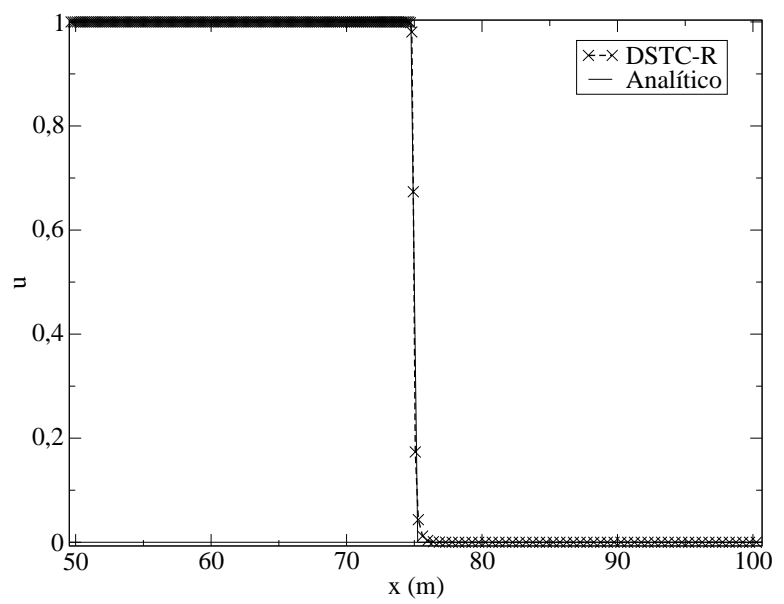

Figura 4: Eq. de Burgers: DSTC-R e solução analítica (malha de 256).

de Darcy). Os seguintes valores numéricos foram utilizados nas simulações: $v=$ $8,11235 e^{-5} ; s_{r_{o}}=0,15 ; s_{r_{w}}=0,20 ; \mu_{o}=10,0 ; \mu_{w}=0,5$. Nos testes feitos para a Eq. de Buckley-Leverett o comprimento da região física foi de $12800 \mathrm{~cm}$ e o tempo total de simulação 300 dias. A condição de contorno em $x=0$ é $f(s)=\lambda_{w}(0,85) v$. O escalar $s$ representa a saturação de água (quantidade de água por cada quantidade de espaço poroso) em uma determinada região em um certo instante do escoamento. A condição inicial é uma saturação de 0,85 para $x \leq 0$ e uma saturação de 0,21 para $x>0$. As grandezas físicas estão expressas em CGS.

As Figuras 5 e 6 apresentam refinamento de malha e a comparação da solução numérica em 128 células com o método NT [6] para o DSTC-R, respectivamente.

Para os testes usando a Eq. de Buckley-Leverett também ocorreu convergência 


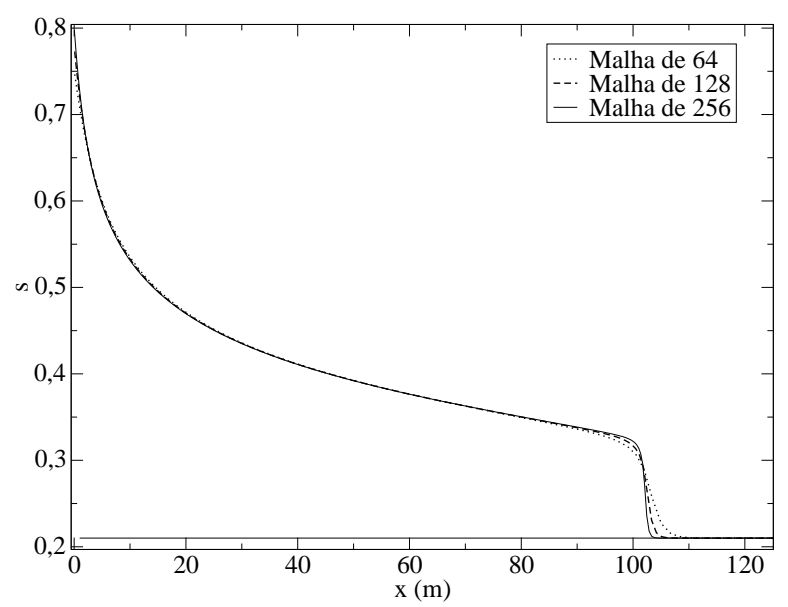

Figura 5: Eq. de Buckley-Leverett: refinamento de malha (DSTC-R).

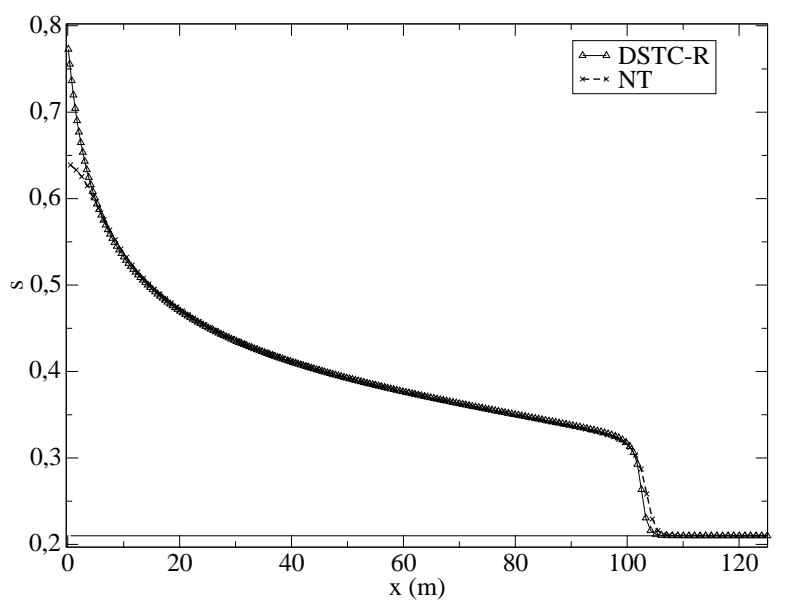

Figura 6: Eq. de Buckley-Leverett: NT e DSTC-R (malha de 128).

numérica sob refinamento da malha computacional. A comparação com o método NT mostra que o choque apresenta velocidade de propagação correta e que o método DSTC-R foi menos difusivo. Além disso, o comportamento na injeção usando o DSTC-R está mais próximo da situação física a ser simulada.

\section{Conclusões}

Neste trabalho foi desenvolvido um método numérico preciso para a solução de leis de conservação escalares em uma dimensão espacial. O método utiliza uma adaptividade da malha computacional para evitar o surgimento de células muito 
reduzidas, que poderiam provocar restrições severas no passo de tempo. A metodologia, denominada DSTC-R resolveu descontinuidades de forma precisa, apresentou baixa difusão numérica, e convergiu, nos experimentos realizados, para a solução fisicamente correta, tanto no caso da Eq. de Burgers (comparação com a solução analítica) como para a Eq. de Buckley-Leverett (comparação com os resultados de [6]). A extensão desta estratégia para problemas bidimensonais está sendo considerada pelos autores.

\title{
Agradecimentos
}

F. Pereira recebeu apoio financeiro dos projetos: CNPq-Edital 05/2004, CT-Petro/ Edital 01/2003, CT-Petro/Edital 016/2005, Edital Institutos do Milênio 01/2005 e Edital 20/2004-Acordo de Cooperação Internacional CNPq/NSF; G. de Souza agradece ao $\mathrm{CNPq}$ (Bolsa de Iniciação Científica) e S. Mancuso agradece à CAPES (Bolsa de Doutorado) pelo apoio.

\begin{abstract}
Efficient methods for convective transport equations are important tools in the computational modeling of engineering problems such as fluid flows in porous media. In this work we present a new numerical method for scalar conservation laws. It uses a lagrangian strategy for the time evolution and grid adaptation. This scheme uses the local conservation identity that appears in $[1,3,4]$. The method was applied in the numerical aproximation of the Burgers and Buckley-Leverett equations (the last one is used in two-phase flow modeling in oil reservoir). The scheme shows good computational perfomance, precision in the capture of the shock and absence of spurious oscillations.
\end{abstract}

\section{Referências}

[1] J. Douglas Jr., F. Pereira, L.M. Yeh, A locally conservative EulerianLagrangian numerical method and its application to nonlinear transport in porous media, Computational Geosciences, 4 (2000), 1-40.

[2] R.J. Le Veque, 'Finite Volume Methods for Hyperbolic Problems", Cambridge Texts in applied Mathematics, Cambridge University Press, UK 2002.

[3] S. Mancuso, "Aproximação numérica de leis de conservação por esquemas euleriano-lagrangeanos localmente conservativos." Dissertação de Mestrado, Instituto Politécnico do Rio de Janeiro - UERJ, 2004.

[4] S. Mancuso, F. Pereira, Esquemas euleriano-lagrangeanos localmente conservativos para leis de conservação hiperbólicas, in "CD-Rom - Iberian Latin American Congress on Computational Methods of Engineering", (2004).

[5] S. Mancuso, F. Pereira, G. de Souza, Um novo método euleriano-lagrangeano para aproximação de leis de conservação, em "Seleta do XXIX CNMAC" (C.F. Bracciali, M.C.C. Cunha, V.L.R. Lopes, H.M. Yang, eds.), TEMA - Tend. Mat. Apl. Comput., Vol. 8, No. 2, pp.277-286, SBMAC, 2007.

[6] N. Nessyahu, E. Tadmor, "Non-oscillatory central differencing scheme for hyperbolic conservation laws", J. Comput. Physics, 87 (1990), 408-463. 